\title{
Frobenius manifolds, integrable hierarchies and minimal Liouville gravity
}

\author{
A.A. Belavin ${ }^{a, c, d}$ and V.A. Belavin ${ }^{b, c}$ \\ ${ }^{a}$ L.D. Landau Institute for Theoretical Physics, \\ 142432 Chernogolovka, Russia \\ ${ }^{b}$ P.N. Lebedev Physical Institute, \\ 119991 Moscow, Russia \\ ${ }^{c}$ Institute for Information Transmission Problems, \\ 127994 Moscow, Russia \\ ${ }^{d}$ Moscow Institute of Physics and Technology, \\ 141700 Dolgoprudny, Russia \\ E-mail: belavin@itp.ac.ru, belavin@lpi.ru
}

ABSTRACT: We use the connection between the Frobrenius manifold and the Douglas string equation to further investigate Minimal Liouville gravity. We search a solution of the Douglas string equation and simultaneously a proper transformation from the KdV to the Liouville frame which ensure the fulfilment of the conformal and fusion selection rules. We find that the desired solution of the string equation has explicit and simple form in the flat coordinates on the Frobenious manifold in the general case of $(p, q)$ Minimal Liouville gravity.

Keywords: Matrix Models, 2D Gravity, Conformal and W Symmetry

ARXIV EPRINT: 1406.6661 


\section{Contents}

1 Introduction 1

2 Frobenius manifolds 2

3 Main example: Frobenius manifold of $A_{q-1}$-type 4

4 Frobenius manifolds and Douglas string equation $\quad 6$

$\begin{array}{ll}4.1 \text { Integrable hierarchies } & 6\end{array}$

$\begin{array}{ll}4.2 \text { Douglas string equation } & 6\end{array}$

$\begin{array}{lll}4.3 & \text { Equation for tau-function } & 7\end{array}$

4.5 $A_{q-1}$ FM and dispersionless limit of Gelfand-Dikij Hierarchy 7

4.6 Formula for tau-function 8

5 Resonance problem in $(p, q)$ MLG $\quad 8$

5.1 Homogeneity property of string equation. Spectrum for $(p, q)$ case 8

5.2 The group of the resonance transformations 9

5.3 The choice of the resonance transformation and of the solution of the string equation 9

6 The plan of the solution of the problem 10

$\begin{array}{lll}7 & \text { Appropriate solution } & 11\end{array}$

8 One-point functions $\quad 12$

9 Two-point functions $\quad 13$

$\begin{array}{ll}10 \text { Conclusions } & 15\end{array}$

\section{Introduction}

The purpose of this paper is to further study Minimal Liouville Gravity (MLG) [1] using an approach based on the Douglas string equation [2]. This study is a continuation of earlier works $[3-6]$.

The Liouville Gravity represents a consistent example of the noncritical String theory. In the initial continuous approach the Liouville Gravity is formulated as a BRST invariant theory composed of the matter sector, the Liouville theory and the ghosts system. MLG represents the theory, where the matter sector is taken to be a $(p, q)$ Minimal Model of CFT [7]. The main problem of MLG is to calculate correlation functions of BRST invariant observables, which are given by integrals over moduli of Riemannian surfaces. Usually 
they are called the correlation numbers. Numerous examples show that the solution of the problem is quite nontrivial within the framework of the continuous approach.

An alternative approach to MLG has grown up from the idea of triangulations of twodimensional surfaces realized in terms of Matrix Models [8-14]. One of the most important points of the approach is the String equation which was derived by Douglas [2] in Matrix Models approach to two dimensional gravity. The subject of the String equation is the generating function of the correlation numbers which depends on the parameters of the problem (the so called KdV times). In our work, following [4-6], we will conjecture that the Douglas equation is applicable to the Minimal Liouville gravity as well as to Matrix Models of 2D gravity.

This conjecture requires the following two questions to be answered: how to choose the desired solution of the Douglas string equation and an appropriate form of the so called resonance transformation [3] from the KdV times to the Liouville coupling constants. Once these two questions are answered, the generating function of the correlation functions in MLG is given explicitly as an integrated one-form defined uniquely for each $(p, q)$ MLG model and coincides with a special choice of the tau-function of the dispersionless limit [15, 16] of the generalized KdV hierarchy.

In this paper, using the connection [5] of the approach to MLG [4] based on the String equation with the Frobenius manifold structure, we find the necessary solution of the String equation. We also show that this solution together with the suitable choosen resonance transformation lead to the results which are consistent with the main requirements of $(p, q)$ models of MLG (the so called selection rules). It is remarkable that the needed solution of the Douglas equation has a very simple form in the flat coordinates on the Frobenious manifold in the general case of $(p, q)$ Minimal Liouville as well as it has been found recently in the case of Unitary models of MLG [6].

The paper is organized as follows. In section 2 we recall briefly the notion of the Frobenius manifold and discuss its basic properties. In section 3 we discuss the Frobenius manifold that appears in the context of Minimal Liouville Gravity. Section 4 is devoted to the connection between the Frobenius manifold structures, Integrable structures and the Douglas string equation. In section 5 we focus on the $(p, q)$ models of MLG and discuss the problem of the resonance transformations. The idea of the approach based on the String equation to $(p, q)$ MLG is formulated in section 6 . The appropriate solution of the Douglas string equation is discussed in section 7 . The rest of the paper is devoted to the analysis of the correlation functions. We show that the special choice of the solution of the String equation together with the resonance transformation encoded in terms of Jacobi polynomials ensure fulfilling the necessary selection rules for the correlation numbers in $(p, q)$ MLG.

\section{Frobenius manifolds}

In this and two next sections we give the definition and a short review of main properties of the Frobenius manifolds needed for our purposes. Here we follow the paper by B.Dubrovin [16], see also [5]. 
By definition a commutative associative algebra $A$ with unity equipped with a nondegenerate invariant bilinear form $($,$) is called Frobenius algebra. The invariance of the$ bilinear form means that for any three vectors $a, b, c$ in $A$ :

$$
(a \cdot b, c)=(a, b \cdot c) .
$$

Let $M$ be $n$-dimensional manifold with a flat metric $\eta_{\alpha \beta} d v^{\alpha} d v^{\beta}$ which is constant in the flat coordinates $v^{\alpha}$.

We introduce in the tangent space $T_{\mathbf{v}} M$ the structure of the Frobenius algebra by the following identification of the bases

$$
\frac{\partial}{\partial v^{\alpha}} \rightarrow e_{\alpha}
$$

Thus, we can multiply tangent vectors at any point of $M$

$$
e_{\alpha} e_{\beta}=C_{\alpha \beta}^{\gamma} e_{\gamma}
$$

The structure constants $C_{\alpha \beta}^{\gamma}$ may depend on $v^{\alpha}$. Such manifold $M$ can be called quasiFrobenius manifold.

Definition 2.1. The manifold $M$ is called Frobenius manifold if these two structures are adjusted with each other in such a way that

(1) the invariant bilinear form $\left(\frac{\partial}{\partial v^{\alpha}}, \frac{\partial}{\partial v^{\beta}}\right)$ is identical to the metric $\eta_{\alpha \beta}$;

(2) the structure of the Frobenius algebra at each point of $M$ and the metric on $M$ are constrainted by the following relation

$$
\nabla_{\rho} C_{\alpha \beta \gamma}=\nabla_{\alpha} C_{\rho \beta \gamma}
$$

The last requirement is equivalent to the requirement that there exists a function $F$ on $M$ which is connected with the structure constants of the Frobenius algebra as

$$
C_{\alpha \beta \gamma}=\frac{\partial^{3} F}{\partial v^{\alpha} \partial v^{\beta} \partial v^{\gamma}},
$$

where

$$
C_{\alpha \beta \gamma}=\eta_{\alpha \rho} C_{\beta \gamma}^{\rho} .
$$

Function $F$ is called Frobenius potential. The consistency of this property with the associativity of the Frobenius algebra is known as WDVV condition [17]

$$
\frac{\partial^{3} F}{\partial v^{\alpha} \partial v^{\beta} \partial v^{\rho}} \eta^{\rho \lambda} \frac{\partial^{3} F}{\partial v^{\lambda} \partial v^{\mu} \partial v^{\nu}}=\frac{\partial^{3} F}{\partial v^{\nu} \partial v^{\beta} \partial v^{\rho}} \eta^{\rho \lambda} \frac{\partial^{3} F}{\partial v^{\lambda} \partial v^{\mu} \partial v^{\alpha}} .
$$

The following statement [16] follows from these properties of the Frobenius manifold $M$. There exist an one-parametric flat deformation $\widetilde{\nabla}_{\alpha}$ of the connection $\nabla_{\alpha}$

$$
\widetilde{\nabla}_{\alpha} x^{\gamma}=\nabla_{\alpha} x^{\gamma}+z C_{\alpha \beta}^{\gamma} x^{\beta},
$$


or, equivalently,

$$
\left[\widetilde{\nabla}_{\alpha}(z), \widetilde{\nabla}_{\beta}(z)\right]=0 .
$$

The proof is based on the associativity of the Frobenius algebra and the equation (2.4). As a consequence of (2.9), there exist $n$ linear independent solutions

$$
\theta^{\alpha}(v, z)=\sum_{k=0}^{\infty} \theta_{k}^{\alpha}(v) z^{k}
$$

of the equation $\widetilde{\nabla}_{\alpha} d \theta^{\lambda}(v, z)=0$, which is equivalent to

$$
\frac{\partial^{2} \theta^{\lambda}}{\partial v^{\alpha} \partial v^{\beta}}(v, z)=z C_{\alpha \beta}^{\gamma} \frac{\partial \theta^{\lambda}}{\partial v^{\gamma}}(v, z)
$$

or

$$
\frac{\partial^{2} \theta_{k+1}^{\lambda}}{\partial v^{\alpha} \partial v^{\beta}}(v)=C_{\alpha \beta}^{\gamma} \frac{\partial \theta_{k}^{\lambda}}{\partial v^{\gamma}}(v)
$$

The functions $\theta^{\alpha}(v, z)$ can be considered as the flat coordinates of the deformed connection $\widetilde{\nabla}_{\alpha}(z)$. We choose $\theta^{\lambda}(v, z)$ so that $\theta^{\lambda}(v, 0)=\theta_{0}^{\lambda}(v)=v^{\lambda}$. From $(2.12)$ it follows, that

$$
\nabla\left(\nabla \theta^{\alpha}\left(v, z_{1}\right), \nabla \theta^{\beta}\left(v, z_{2}\right)\right)=\left(z_{1}+z_{2}\right) \nabla \theta^{\alpha}\left(v, z_{1}\right) \cdot \nabla \theta^{\beta}\left(v, z_{2}\right)
$$

and, hence, the scalar product $\left(\nabla \theta^{\alpha}(v, z), \nabla \theta^{\beta}(v,-z)\right)=$ Const $(z)$ does not depend on $v^{\alpha}$. For $z=0$ we find $\operatorname{Const}(0)=\eta^{\alpha \beta}$. Equation (2.12) is invariant with respect to the transformation

$$
\theta^{\mu}(v, z) \rightarrow A_{\nu}^{\mu}(z) \theta^{\nu}(v, z)
$$

where $A_{\nu}^{\mu}(0)=\delta_{\nu}^{\mu}$. Using these transformations one can fix the normalization in such a way that

$$
\left(\nabla \theta^{\alpha}(v, z), \nabla \theta^{\beta}(v,-z)\right)=\eta^{\alpha \beta}
$$

\section{Main example: Frobenius manifold of $A_{q-1}$-type}

Our main example is $A_{q-1}$ Frobenius manifold [17]. Let $Q(y)$ be a polynomial of $y$

$$
Q(y)=y^{q}+u_{1} y^{q-2}+\ldots+u_{q-1},
$$

and $\left\{u_{\alpha}\right\}$ represent some coordinates on $M$. We call $\left\{u_{\alpha}\right\}$ the canonical coordinates.

Definition 3.1. $A_{q-1}$ Frobenius algebra is the space of polynomials modulo polynomial $\frac{d Q}{d y}$ :

$$
A_{q-1}(u)=\mathbb{C}[y] / \frac{d Q}{d y} .
$$

The corresponding manifold $M$ is called the Frobenius manifold of $A_{q-1}$ type 
The polynomials

$$
P_{\alpha}(y)=\frac{\partial Q}{\partial u_{\alpha}},
$$

form a basis in the tangent space $T_{\mathbf{v}} M$. An invariant bilinear form (which is equivalent to the metric) is defined by

$$
\left(P_{\alpha}, P_{\beta}\right)=\underset{y=\infty}{\operatorname{res}}\left(\frac{P_{\alpha}(y) P_{\beta}(y)}{\frac{d Q}{d y}(y)}\right) .
$$

With this definition one can verify that the corresponding metric is flat and

$$
C_{\alpha \beta \gamma}=\nabla_{\alpha} \nabla_{\beta} \nabla_{\gamma} F(u)
$$

To this end we perform the transformation from the canonical coordinates $\left\{u_{\alpha}\right\}$ to the new coordinates $\left\{v^{\alpha}\right\}$ by means of the following relation

$$
y=z-\frac{1}{q}\left(\frac{v^{q-1}}{z}+\frac{v^{q-2}}{z^{2}}+\cdots+\frac{v^{1}}{z^{q-1}}\right)+\mathcal{O}\left(\frac{1}{z^{q+1}}\right),
$$

where $z^{q}=Q(y)$.

Some useful properties of the new coordinates are formulated in the following

Theorem 3.2. From the transformation (3.6) it follows that

1. $v^{\alpha}$ form flat coordinates, i.e., the metric in this coordinates is constant and

$$
\eta_{\alpha \beta}=-q\left(\frac{\partial Q}{\partial v^{\alpha}}, \frac{\partial Q}{\partial v^{\beta}}\right)=\delta_{\alpha+\beta, q}
$$

2 .

$$
C_{\alpha \beta \gamma}=-q \underset{y=\infty}{\operatorname{res}}\left(\frac{\frac{\partial Q}{\partial v^{\alpha}} \frac{\partial Q}{\partial v^{\beta}} \frac{\partial Q}{\partial v^{\gamma}}}{\frac{d Q}{d y}}\right)=\frac{\partial^{3} F}{\partial v^{\alpha} \partial v^{\beta} \partial v^{\gamma}} .
$$

3.

$$
\theta_{\alpha, k}=-c_{\alpha, k} \underset{y=\infty}{\operatorname{res}} Q^{k+\frac{\alpha}{q}}(y)
$$

where

$$
c_{\alpha, k}=\frac{\Gamma\left(\frac{\alpha}{q}\right)}{\Gamma\left(\frac{\alpha}{q}+k+1\right)} .
$$

To prove these statements it is convenient to use the basis elements of $A_{q-1}$ in flat coordinates defined by $\Phi_{\alpha}(y)=\frac{\partial Q(y)}{\partial v^{\alpha}}$ which possess the following property

$$
\Phi_{\alpha}(y)=\frac{1}{\alpha} \frac{d}{d y}\left(Q^{\frac{\alpha}{q}}\right)_{+} .
$$

In what follows we use the following convention

$$
\theta_{\mu, k}=\theta_{\mu-q\lfloor\mu / q\rfloor, k+\lfloor\mu / q\rfloor},
$$

where $\lfloor\mu / q\rfloor$ is the integer part of $\mu / q$. It is clear that (3.12) agrees with the definition (3.9). 


\section{Frobenius manifolds and Douglas string equation}

\subsection{Integrable hierarchies}

Let $\mathcal{M}$ be a space of functions of $x$ taking values in $M$. Let $I$ and $J$ be functionals on $\mathcal{M}$. We define the Poisson bracket on $\mathcal{M}$ as

$$
\{I, J\}=\int \frac{\delta I}{\delta v^{\alpha}(x)} \eta^{\alpha \beta} \frac{d}{d x} \frac{\delta I}{\delta v^{\beta}(x)} d x,
$$

or

$$
\left\{v^{\alpha}(x), v^{\beta}(y)\right\}=\eta^{\alpha \beta} \delta^{\prime}(x-y),
$$

where, as usual in the calculus of variations, the integrand is defined modulo total derivatives. The functionals

$$
H_{\alpha, k}=\int \theta_{\alpha, k+1}(\vec{v}(x)) d x, \quad \alpha=1, \ldots, n, \quad k \geq 0,
$$

mutually commute among themselves

$$
\left\{H_{\alpha, k}, H_{\beta, l}\right\}=0 \text {. }
$$

As a result, the Hamiltonian flows

$$
\frac{\partial v^{\mu}}{\partial t_{k}^{\alpha}}=\left\{v^{\mu}, H_{\alpha, k}\right\}=\eta^{\mu \nu} \frac{\partial}{\partial x} \frac{\partial \theta_{\alpha, k+1}}{\partial v^{\nu}}=C_{\lambda}^{\mu \rho} \frac{\partial \theta_{\alpha, k}}{\partial v^{\rho}} \frac{\partial v^{\lambda}}{\partial x} .
$$

commute, i.e.,

$$
\frac{\partial}{\partial t_{l}^{\beta}} \frac{\partial \vec{v}}{\partial t_{k}^{\alpha}}=\frac{\partial}{\partial t_{k}^{\alpha}} \frac{\partial \vec{v}}{\partial t_{l}^{\beta}}
$$

It follows from (4.5) that $t_{0}^{1}=x$.

\subsection{Douglas string equation}

Let us define a function $S(v, t)$ on $M$ which depends on the additional papametres $\left\{t_{k}^{\alpha}\right\}$

$$
S\left(v, t_{k}^{\alpha}\right)=\sum_{\alpha=1}^{n} \sum_{k \geq 0} t_{k}^{\alpha} \theta_{\alpha, k}(v) .
$$

The equation

$$
\frac{\partial S}{\partial v^{\alpha}}=0
$$

is called a string equation. In the case of Frobenius manifold of $A_{q-1}$ type it is nothing but the Douglas string equation written in the form of the principle of least String action [19]. It can be shown that solutions $\vec{v}\left(t_{k}^{\alpha}\right)$ of the string equation (4.8) satisfy also (4.5). 


\subsection{Equation for tau-function}

We define the function $Z[t]=\log \tau(t)$, where

$$
Z[t]=\frac{1}{2} \int_{0}^{v=v^{*}(t)} \Omega
$$

and

$$
\Omega=C_{\alpha}^{\beta \gamma}(v) \frac{\partial S(v, t)}{\partial v^{\beta}} \frac{\partial S(v, t)}{\partial v^{\gamma}} d v^{\alpha},
$$

is the differential form and $v^{*}(t)$ is one of the solutions of the string equation (4.8). From the associativity of the algebra $A_{q-1}$ and the equations (2.12) it follows that $\Omega$ is closed one-form.

Lemma 4.4. $Z(t)$ satisfies

$$
\frac{\partial^{2} Z(t)}{\partial t_{k}^{\alpha} \partial t_{0}^{1}}=\theta_{\alpha, k}(v(t))
$$

In particular,

$$
v^{\alpha}(t)=\eta^{\alpha \beta} \frac{\partial^{2} Z}{\partial t_{0}^{\beta} \partial t_{0}^{1}}
$$

and for $v^{q-1}(t)=u_{1}(t)$

$$
\frac{\partial^{2} Z}{\partial x^{2}}=u_{1}(t)
$$

Proof. Differentiating with respect to $t_{k}^{\alpha}$ and $t_{0}^{1}$ and taking into account the string equation, we find

$$
\frac{\partial^{2} Z}{\partial t_{k}^{\alpha} \partial t_{0}^{1}}=\int_{0}^{v^{*}(t)} C_{\lambda}^{\beta \gamma} \frac{\partial \theta_{\alpha, k}}{\partial v^{\beta}} \cdot \frac{\partial \theta_{1,0}}{\partial v^{\gamma}} d v^{\lambda}=\int_{0}^{v^{*}(t)} \frac{\partial \theta_{\alpha, k}}{\partial v^{\lambda}} d v^{\lambda}=\theta_{\alpha, k} .
$$

Here we used that $\theta_{1,0}=v_{1}=v^{q-1}, C_{\lambda}^{\beta, q-1}=\delta_{\lambda}^{\beta}$.

Taking into account $\theta_{\alpha, 0}=v_{\alpha}$ we obtain from Lemma 4.4 that

$$
\frac{\partial^{2} Z(t)}{\partial t_{0}^{\alpha} \partial t_{0}^{1}}=v_{\alpha}
$$

Since $Z$ satisfy equations (4.8) and (4.13), it is a tau-function of the integrable hierarchy connected with the corresponding Frobenius manifold.

\section{5 $A_{q-1}$ FM and dispersionless limit of Gelfand-Dikij Hierarchy}

The dispersionless limit of the Gelfand-Dikij equations is formulated as follows:

$$
\frac{\partial Q}{\partial t_{k}^{\alpha}}=\left[A_{\alpha, k}, Q\right]=\frac{\partial A_{\alpha, k}}{\partial x} \frac{\partial Q}{\partial y}-\frac{\partial A_{\alpha, k}}{\partial y} \frac{\partial Q}{\partial x},
$$

where

$$
Q=y^{q}+u_{1}(x) y^{q-2}+\ldots+u_{q-1},
$$


and

$$
A_{\alpha, k}=\frac{1}{q} c_{\alpha, k}\left(Q^{k+\frac{\alpha}{q}}\right)_{+}
$$

One can show that these equations are equivalent to the Hamiltonian equation

$$
\frac{\partial v^{\mu}}{\partial t_{k}^{\alpha}}=\eta^{\mu \nu} \frac{\partial}{\partial x} \frac{\partial \theta_{\alpha, k+1}}{\partial v^{\nu}}
$$

\subsection{Formula for tau-function}

As it was derived above the logarithm of the tau-function $Z\left[\left\{t_{k}^{\alpha}\right\}\right]$ is given by

$$
Z\left[\left\{t_{k}^{\alpha}\right\}\right]=\frac{1}{2} \int_{0}^{v^{*}(t)} C_{\alpha}^{\beta \gamma} \frac{\partial S}{\partial v^{\beta}} \frac{\partial S}{\partial v^{\gamma}} d v^{\alpha},
$$

where $v^{*}(t)$ is the solution of the string equation

$$
\left.\frac{\partial S}{\partial v^{\alpha}}\right|_{v^{\alpha}=v^{* \alpha(t)}}
$$

and

$$
S=\sum_{\alpha=1}^{q-1} \sum_{k} t_{k}^{\alpha} \theta_{\alpha, k}
$$

\section{Resonance problem in $(p, q)$ MLG}

\subsection{Homogeneity property of string equation. Spectrum for $(p, q)$ case}

Let now only the finite number of the parameters $\left\{t_{k}^{\alpha}\right\}$ be nonzero. One of them we take equal to one and others be enumerated by two integers $(m, n)$. Here $1 \leq m \leq q-1$, $1 \leq n \leq p-1$, where $p, q$ are two coprime integers, $p>q$ and $q$ is a degree of the polynomial $Q$ defined in (4.17). Hence, the set of the parameters $\left\{t_{k}^{\alpha}\right\}$ is replaced by the set $\left\{\tau_{m n}\right\}$. Let us take the action in the form

$$
S=\underset{y=\infty}{\operatorname{res}}\left[Q^{\frac{p+q}{q}}+\sum_{m, n}^{p m-q n>0} \tau_{m n} Q^{\frac{p m-q n}{q}}\right],
$$

It is easy to check that $Q\left[y, u_{\alpha}\right]$ and $S\left[u_{\alpha}, \tau_{m n}\right]$ are quasi-homogeneous functions

$$
Q\left[\rho y, \rho^{r_{\alpha}} u_{\alpha}\right]=\rho^{q} Q\left[y, u_{\alpha}\right], \quad S\left[\rho^{r_{\alpha}} u_{\alpha}, \rho^{\sigma_{m n}} \tau_{m n}\right]=\rho^{p} S\left[u_{\alpha}, \tau_{m n}\right] .
$$

Here we denote

$$
r_{\alpha}=q-\alpha-1, \quad \sigma_{m n}=p+q-|p m-q n| .
$$

We call $\left\{\sigma_{m n}\right\}$ the set of the scaling indices of the set $\left\{\tau_{m n}\right\}$. As it was found by Douglas [2], the numbers $\delta_{m n}=\frac{\sigma_{m n}}{2 q}$ coincide with the gravitational dimensions of the physical fields in $(p, q)$ Minimal Liouvillle gravity [18].

The function $Z\left[\tau_{m n}\right]$ is a quasi-homogeneous function

$$
Z\left[\rho^{2 q \delta_{m n}} \tau_{m n}\right]=\rho^{p+q} Z\left[\tau_{m n}\right] .
$$




\subsection{The group of the resonance transformations}

Since the scaling indices are integer, the following relation can take place

$$
\sigma_{m n}=\sigma_{k_{1} l_{1}}+\sigma_{k_{2} l_{2}}+\ldots+\sigma_{k_{N} l_{N}}
$$

This is known as a resonance condition. The number of possible resonances grows when $p$ and $q$ increase. A transformation $\tau_{m n} \rightarrow \lambda_{m n}$ of the form

$\tau_{m n}=\lambda_{m n}+\sum_{k_{1}, l_{1}, k_{2}, l_{2}} A_{m n}^{k_{1} l_{1} ; k_{2}, l_{2}} \lambda_{k_{1}, l_{1}} \lambda_{k_{2}, l_{2}}+\sum_{k_{1}, l_{1}, k_{2}, l_{2}, k_{3}, l_{3}} A_{m n}^{k_{1} l_{1} ; k_{2}, l_{2} ; k_{3}, l_{3}} \lambda_{k_{1}, l_{1}} \lambda_{k_{2}, l_{2}} \lambda_{k_{3}, l_{3}}+\ldots$

is called resonance transformation if (5.5) is satisfied for each term. Besides, by definition, we suggest that the scaling index of $\lambda_{m n}$ equals to the one of $\tau_{m n}$.

It is obvious that

$$
\tau_{m n}\left(\left\{\rho^{\sigma_{k l}} \lambda_{k l}\right\}\right)=\rho^{\sigma_{m n}} \tau_{m n}\left(\left\{\lambda_{k l}\right\}\right),
$$

and that the resonance transformation does not change the homogeneity property of the partition function $Z\left[\tau_{m n}\left(\left\{\lambda_{k l}\right\}\right)\right]=\widetilde{Z}\left[\lambda_{m n}\right]$

$$
\widetilde{Z}\left[\left\{\rho^{\sigma_{m n}} \lambda_{m n}\right\}\right]=\rho^{p+q} \widetilde{Z}\left[\left\{\lambda_{m n}\right\}\right] .
$$

Hence, if we find some solution of the string equation (4.8) and construct $Z\left[\tau_{m n}\right]$, then we get a family of the solutions $\widetilde{Z}\left[\left\{\lambda_{m n}\right\}\right]=Z\left[\left\{\tau_{m n}\left(\left\{\lambda_{k l}\right)\right\}\right\}\right]$ having the same homogeneity properties with respect to the resonance transformations.

\subsection{The choice of the resonance transformation and of the solution of the string equation}

Now we are in the position to formulate the following problem. We are looking for solutions of the string equation and resonance transformations which gives function $\widetilde{Z}\left[\left\{\lambda_{m n}\right\}\right]$ satisfying infinite number of constraints known as fusion rules for observables of minimal CFT models $M(p, q)$ and their for the correlators. In what follows we restrict ourself by considering spherical topology. Then these rules can be formulated as follows.

We denote by $\Phi_{m n}$, where $1 \leq m \leq p$ and $1 \leq n \leq q$, the primary fields in the minimal model $M(p, q)$ of Conformal field theory. The fields $\Phi_{m, n}$ and $\Phi_{q-m, p-n}$ correspond to the same primary field.

The following graphical representation allows to formulate these restrictions

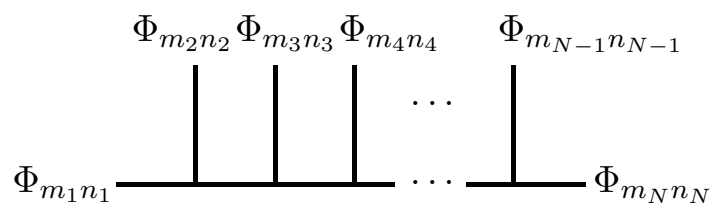

Here the external lines represent the (arbitrary arranged) primary fields in the correlator $\left\langle\Phi_{m_{1} n_{1}} \Phi_{m_{2} n_{2}} \ldots \Phi_{m_{N} n_{N}}\right\rangle$ (here we assume $N \geq 3$ ). The fusion rules result to the requirement that the correlation function must be equal to zero if there are no sets of pairs $\left(k_{i}, l_{i}\right)$ 
assigned to the internal lines, for which in any vertex of the graph the following condition on the three pairs $\left(m_{i}, n_{i}\right)(i=1,2,3)$ corresponding to the lines connected to this vertex

$$
\begin{gathered}
\left|m_{1}-m_{2}\right|+1 \leq m_{3} \leq \min \left\{m_{1}+m_{2}-1,2 q+1-m_{1}-m_{2}\right\} \quad \text { step } 2, \\
\left|n_{1}-n_{2}\right|+1 \leq n_{3} \leq \min \left\{n_{1}+n_{2}-1,2 p+1-n_{1}-n_{2}\right\} \quad \text { step 2, }
\end{gathered}
$$

can not be satisfied any permutation of the pairs.

In addition, from the conformal selection rules for $N=1$ it follows

$$
\left\langle\Phi_{m n}\right\rangle=0
$$

for $(m, n) \neq(1,1)$ and for $N=2$

$$
\left\langle\Phi_{m_{1} n_{1}} \Phi_{m_{2} n_{2}}\right\rangle=0
$$

for $\left(m_{1}, n_{1}\right)$ not equal to $\left(m_{2}, n_{2}\right)$ or $\left(q-m_{2}, p-n_{2}\right)$.

Now we are going to give a more precise formulation of our main conjecture.

Conjecture 5.4. There exist the solution of the string equation and the choice of the resonance transformation described above, such that the function

$$
\widetilde{Z}\left[\left\{\lambda_{m n}\right\}\right]=\left\langle\exp \sum_{m, n} \lambda_{m, n} O_{m, n}\right\rangle=\sum_{N=0}^{\infty} \sum_{m_{i}, n_{i}} \frac{\lambda_{m_{1} n_{1}} \ldots \lambda_{m_{N} n_{N}}}{N !}\left\langle O_{m_{1} n_{1}} \ldots O_{m_{N} n_{N}}\right\rangle,
$$

appeares the generating function of the correlators in the Minimal Liouville Gravity. vanish.

In particular, all correlators $\left\langle O_{m_{1} n_{1}} \ldots O_{m_{N} n_{N}}\right\rangle$ forbidden by the conformal fusion rules

\section{The plan of the solution of the problem}

To solve the formulated above problem we write the action $S\left(v_{\alpha}, t_{m n}\right)$ and the generating function $Z\left[\left\{t_{m n}\right\}\right]$ in terms of new variables $\left\{\lambda_{m n}\right\}$ using the resonance change of variables

$$
\begin{aligned}
t_{m n}= & \lambda_{m n}+A_{m n} \mu^{\delta_{m n}}+\sum_{m_{1}, n_{1}}^{\delta_{m_{1} n_{1}} \leq \delta_{m n}} A_{m n}^{m_{1} n_{1}} \mu^{\delta_{m n}-\delta_{m_{1} n_{1}}} \lambda_{m_{1} n_{1}}+ \\
& \delta_{m_{1} n_{1}+\delta_{m_{2} n_{2}} \leq \delta_{m n}}^{\delta_{m}} A_{m n}^{m_{1} n_{1}, m_{2} n_{2}} \mu^{\delta_{m n}-\delta_{m_{1} n_{1}}-\delta_{m_{2} n_{2}}} \lambda_{m_{1} n_{1}} \lambda_{m_{2} n_{2}}+\ldots,
\end{aligned}
$$

where $\mu=\lambda_{11}$ is called the cosmological constant in the continuum approach to MLG.

After performing this transform the action takes the form

$$
\begin{aligned}
\widetilde{S}\left[v_{\alpha},\left\{\lambda_{m n}\right\}\right]= & S^{(0)}\left[v_{\alpha}\right]+\sum_{m, n} \lambda_{m n} S^{(m n)}\left(v_{\alpha}\right)+ \\
& +\sum_{m_{1}, n_{1}, m_{2}, n_{2}} \lambda_{m_{1} n_{1}} \lambda_{m_{2} n_{2}} S^{\left(m_{1} n_{1}, m_{2} n_{2}\right)}\left(v_{\alpha}\right)+\ldots
\end{aligned}
$$


The information about the form of the resonance transformation is encoded in the coefficients of $S^{(0)}, S^{(m n)}$, etc. From (3.9) and (12.1) we find

$$
\begin{aligned}
S^{(0)} & =\underset{y=\infty}{\operatorname{res}}\left[Q^{\frac{p+q}{q}}+\sum_{l=1}^{s} A_{1 n} \mu^{\frac{l+1}{2}} Q^{\frac{p-q l}{q}}\right], \\
S^{(m n)} & =\underset{y=\infty}{\operatorname{res}}\left[Q^{\frac{p m-q n}{q}}+\sum_{l=n+2}^{s m} A_{m l}^{m n} \mu^{\frac{l-n}{2}} Q^{\frac{p m-q l}{q}}\right],
\end{aligned}
$$

where $A_{k l}^{m n}$ are the coefficients of the resonance relations. The higher coefficients can also be easily written in terms of the coefficients $A_{k l}^{\left\{m_{i} n_{i}\right\}}$.

The generating function is given by

$$
\widetilde{Z}\left[\left\{\lambda_{m n}\right\}\right]=\frac{1}{2} \int_{0}^{\mathbf{v}^{*}} C_{\alpha}^{\beta \gamma}(v) \frac{\partial \widetilde{S}}{\partial v^{\beta}} \frac{\partial \widetilde{S}}{\partial v^{\gamma}} d v^{\alpha}
$$

where $\mathbf{v}^{*}$ is defined as a function of the parameters $\left\{\lambda_{m n}\right\}$ of the Douglas string equation (4.8).

From now on we will skip the tilde over the functions $\widetilde{S}\left(\left\{u_{\alpha}\right\},\left\{\lambda_{m n}\right\}\right)$ and $\widetilde{Z}\left(\left\{\lambda_{m n}\right\}\right)$.

\section{Appropriate solution}

To compute the one-point function which is given by the integral

$$
\left\langle O_{m n}\right\rangle=\int_{0}^{v_{\alpha}^{0}} C_{\beta \gamma}^{\alpha} \frac{\partial S^{(0)}}{\partial v_{\beta}} \frac{\partial S^{(m n)}}{\partial v_{\gamma}} d v_{\alpha}
$$

we need to know the upper limit in this integral $v_{\alpha}^{0}$ which is the solution of the string equation for all couplings (except $\lambda_{11}=\mu$ ) equal to zero

$$
v_{\alpha}^{0}=\left.v_{\alpha}^{*}\left(\lambda_{m n}\right)\right|_{\lambda_{m n}=0, \lambda_{11}=\mu} .
$$

Explicitly, $v_{\alpha}^{0}$ satisfies

$$
\left.\frac{\partial S^{(0)}}{\partial v_{\mu}}\right|_{v_{\alpha}=v_{\alpha}^{0}}=0 .
$$

We introduce the new positive integer numbers $\mathrm{s}$ and $p_{0}$ such that $p=s q+p_{0}$ and $0<$ $p_{0}<q$. Using (6.3), (6.4) and (3.9), $S^{(0)}$ and $S^{(m n)}$ can be written as

$$
\begin{aligned}
S^{(0)} & =-\frac{\theta_{p_{0}, s+1}}{c_{p_{0}, s+1}}-\sum_{l=1}^{s} A_{1 l} \mu^{\frac{l+1}{2}} \frac{\theta_{p_{0}, s-l}}{c_{p_{0}, s-l}} \\
S^{(m n)} & =-\frac{\theta_{p_{0} m, s m-n}}{c_{p_{0} m, s m-n}}-\sum_{l=n+2}^{s m} A_{m l}^{m n} \mu^{\frac{l+1}{2}} \frac{\theta_{p_{0} m, s m-l}}{c_{p_{0} m, s m-l}} .
\end{aligned}
$$

We will use the following proposition from [6]: 
Proposition 7.1. On the line $v_{i>1}=0$,

$$
\begin{cases}k \text { even }: & \frac{\partial \theta_{\lambda, k}}{\partial v_{\alpha}}=\delta_{\lambda, \alpha} x_{\lambda, k}\left(-\frac{v_{1}}{q}\right)^{\frac{k}{2} q} \\ k \text { odd }: & \frac{\partial \theta_{\lambda, k}}{\partial v_{\alpha}}=\delta_{\lambda, q-\alpha} y_{\lambda, k}\left(-\frac{v_{1}}{q}\right)^{\frac{k-1}{2} q+\lambda}\end{cases}
$$

where

$$
x_{\alpha, k}=\frac{\Gamma\left(\frac{\alpha}{q}\right)}{\Gamma\left(\frac{\alpha}{q}+\frac{k}{2}\right)\left(\frac{k}{2}\right) !} \quad \text { and } \quad y_{\lambda, k}=-\frac{\Gamma\left(\frac{\alpha}{q}\right)}{\Gamma\left(\frac{\alpha}{q}+\frac{k+1}{2}\right)\left(\frac{k-1}{2}\right) !} .
$$

Using this statement together with (7.4) it is not difficult to see that the string equation (7.3) has the solutions of the form $v_{\alpha}^{0}=0$ for $\alpha \neq 1$ and the coordinate $v_{1}^{0}$ is a root of the equation

$$
\frac{\partial S^{(0)}}{\partial v_{p_{0}}}=0, \quad \text { if } \quad s-\text { odd }
$$

or

$$
\frac{\partial S^{(0)}}{\partial v_{q-p_{0}}}=0, \quad \text { if } \quad s-\text { even. }
$$

Here we assume that after taking derivative we set all $v_{\alpha}$ for $\alpha \neq 1$ to zero. More explicitly these equations can be written as

$$
\frac{\partial S_{0}}{\partial v_{p_{0}}}=\sum_{k=-1: 2: p-1} B_{p_{0}, k}^{\text {odd }}\left(-\frac{v_{1}}{q}\right)^{\frac{s-k}{2} q}, \quad \text { if } \quad s-\text { odd },
$$

or

$$
\frac{\partial S_{0}}{\partial v_{q-p_{0}}}=\sum_{k=-1: 2: p-1} B_{p_{0}, k}^{\text {odd }}\left(-\frac{v_{1}}{q}\right)^{\frac{s-k-1}{2} q}, \quad \text { if } \quad s-\text { even, }
$$

where

$$
B_{p_{0}, k}^{\text {odd }}=\frac{x_{p_{0}, k}}{c_{p_{0}, k}} A_{1, k} \mu^{\frac{k+1}{2}}
$$

and

$$
B_{p_{0}, k}^{\text {even }}=\frac{y_{p_{0}, k}}{c_{p_{0}, k}} A_{1, k} \mu^{\frac{k+1}{2}}
$$

where $A_{1,-1}=1$.

\section{One-point functions}

As it was shown in [6], the structure constant in the flat coordinates on the line $v_{\alpha>0}=0$, for $\alpha \geq \beta \geq \gamma$

$$
C_{\alpha \beta \gamma}=\Theta_{1, q-1}(\alpha+\beta-\gamma)\left(-\frac{v_{1}}{q}\right)^{\frac{\alpha+\beta+\gamma-q-1}{2}} \text { if } \frac{\alpha+\beta+\gamma-q-1}{2} \in \mathbb{N}_{0} \text {, otherwise 0, }
$$

where $\mathbb{N}_{0}$ is the set of non-negative integers. Here $\Theta_{A, B}(x)=1$ if $x \in[A, B]$ and is zero otherwise. Using (7.6) we find for $s$ odd and $(s m-n)$ even

$$
\left\langle O_{m n}\right\rangle=\int_{0}^{v_{1}^{0}} C_{q-1, p_{0}, p_{0} m} \frac{\partial S^{(0)}}{\partial v_{p_{0}}} \frac{\partial S^{(m n)}}{\partial v_{p_{0} m}} d v_{1} .
$$


Taking into account (8.1) we conclude that the correlation function is zero for $m \neq 1$. Hence, in this case from the selection rules we obtain

$$
\left\langle O_{1 n}\right\rangle=\int_{0}^{v_{1}^{0}}\left(-\frac{v_{1}}{q}\right)^{p_{0}-1} \frac{\partial S^{(0)}}{\partial v_{p_{0}}} \frac{\partial S^{(1 n)}}{\partial v_{p_{0}}} d v_{1}=0 .
$$

For $s$ odd and $(s m-n)$ odd, the gravitational dimension

$$
\left[\left\langle O_{m n}\right\rangle\right]=\frac{p+q}{q}-\delta_{m n}=\frac{s m-n}{2}+\frac{s+1}{2}+\frac{p_{0} m+p_{0}}{2 q},
$$

is integer, the correlation function is analytic and, therefore, should not be considered [5]. Similarly, for $s$ even and $(s m-n)$ even, we obtain the following consequence of the selection rules

$$
\left\langle O_{1 n}\right\rangle=\int_{0}^{v_{1}^{0}}\left(-\frac{v_{1}}{q}\right)^{q-p_{0}-1} \frac{\partial S^{(0)}}{\partial v_{q-p_{0}}} \frac{\partial S^{(1 n)}}{\partial v_{q-p_{0}}} d v_{1}=0 .
$$

Finally, if $s$ even and $(s m-n)$ odd, we find again that the expressions for the one point correlation functions are analytic.

Simple analysis shows that the number of these equations is equal to the number of the coefficients arising in the first order in the resonance relation. Hence the requirement of absence of the one point functions fixes uniquely unknown coefficients $B_{p_{0} k}$ in the expressions (7.10) and (7.11).

Thus we arrive to the conclusion that the special solution of the string equation considered above ensure the requirements of the selection rules in agreement with the general prescription described in the previous section.

We note also that the variety of $(p, q)$ models of minimal Liouville Gravity is splitted in two subclasses according to the condition that $\lfloor p / q\rfloor$ be either even or odd. In each case we find distinct sets of requirements formulated above leading to zero valued one point functions.

\section{Two-point functions}

We are now going to consider the two-point function. From (6.5) we find

$$
\left\langle O_{m_{1} n_{1}} O_{m_{2} n_{2}}\right\rangle=\sum_{\gamma=1}^{q-1}(-q)^{1-\gamma} \int_{0}^{v_{1}^{0}} d v_{1}\left(-\frac{v_{1}}{q}\right)^{\gamma-1} \frac{\partial S^{\left(m_{1} n_{1}\right)}}{\partial v_{\gamma}} \frac{\partial S^{\left(m_{2} n_{2}\right)}}{\partial v_{\gamma}} .
$$

It follows from (7.6) that $\frac{\partial S^{(m n)}}{\partial v^{\gamma}} \neq 0$ if one of the following two conditions is satisfied

$$
\begin{aligned}
& \text { 1) } \gamma=m p_{0} \bmod q \quad \text { and }(s m-n)-\text { even, } \\
& \text { 2) } \gamma=q-m p_{0} \bmod q \text { and }(s m-n)-\text { odd. }
\end{aligned}
$$

Similarly to the consideration in the previous section we find four cases where the two point function can be non-zero. In two cases: where the first pair $\left(m_{1}, n_{1}\right)$ satisfies first condition while the second pair $\left(m_{2}, n_{2}\right)$ is subject of the second condition and vice versa, 
we find the regular expression for the two point function. Thus, we are left with the two options where both pairs satisfy either the first or the second condition in (9.2).

Explicitly, in the case when both $\left(s m-n_{1}\right)$ and $\left(s m-n_{2}\right)$ are even we get the following requirement

$$
\left\langle O_{m n_{1}} O_{m n_{2}}\right\rangle=\int_{0}^{v_{1}^{0}} d v_{1}\left(-\frac{v_{1}}{q}\right)^{m p_{0}-1} \frac{\partial S^{\left(m n_{1}\right)}}{\partial v_{m p_{0}}} \frac{\partial S^{\left(m n_{2}\right)}}{\partial v_{m p_{0}}}=0 \quad \text { if } \quad n_{1} \neq n_{2} .
$$

Making the substitution

$$
t=2\left(\frac{v_{1}}{v_{1}^{0}}\right)^{q}-1
$$

and denoting

$$
\frac{\partial S^{(m n)}}{\partial v_{m p_{0}}}=L_{\frac{s m-n}{2}}(t)
$$

we find the following consequence of the diagonality condition

$$
\left\langle O_{m n_{1}} O_{m n_{2}}\right\rangle=\int_{-1}^{1} d t(1+t)^{\frac{m p_{0}-q}{q}} L_{\frac{s m-n_{1}}{2}}(t) L_{\frac{s m-n_{2}}{2}}(t)=0 \quad \text { if } \quad n_{1} \neq n_{2} .
$$

Hence, the selection rules for the two-point correlation numbers requires that the polynomials $\frac{L_{\frac{s m-n}{2}}}{2}$ form an orthogonal set of Jacobi polynomials

$$
\frac{\partial S^{(m n)}}{\partial v_{m p_{0}}}=\frac{p m-q n}{q} P_{\frac{s m-n}{2}}^{\left(0, \frac{m p_{0}-q}{q}\right)}(t), \quad \text { for } \quad(s m-n)-\text { even. }
$$

In the second case, where both $\left(s m-n_{1}\right)$ and $\left(s m-n_{2}\right)$ are odd, we have

$$
\left\langle O_{m n_{1}} O_{m n_{2}}\right\rangle=\int_{0}^{v_{1}^{0}} d v_{1}\left(-\frac{v_{1}}{q}\right)^{q-m p_{0}-1} \frac{\partial S^{\left(m n_{1}\right)}}{\partial v_{q-m p_{0}}} \frac{\partial S^{\left(m n_{2}\right)}}{\partial v_{q-m p_{0}}}=0 \quad \text { if } \quad n_{1} \neq n_{2} .
$$

Denoting

$$
\frac{\partial S^{(m n)}}{\partial v_{q-m p_{0}}}=(1+t)^{\frac{m p_{0}}{q}} L_{\frac{s m-n-1}{2}}(t)
$$

we find the following consequence of the diagonality condition for the two-point correlation function in this case

$$
\left\langle O_{m n_{1}} O_{m n_{2}}\right\rangle=\int_{-1}^{1} d t(1+t)^{\frac{m p_{0}}{q}} L_{\frac{s m-n_{1}-1}{2}}(t) L_{\frac{s m-n_{2}-1}{2}}(t)=0 \quad \text { if } \quad n_{1} \neq n_{2} .
$$

It means that

$$
\frac{\partial S^{(m n)}}{\partial v_{q-m p_{0}}}=\frac{p m-q n}{q}(1+t)^{\frac{m p_{0}}{q}} P_{\frac{s m-n-1}{2}}^{\left(0, \frac{m p_{0}}{q}\right)}(t) \quad \text { for } \quad(s m-n)-\text { odd. }
$$

At last, inserting these explicit expressions for the derivatives of $S^{(m n)}$ to the equations (8.3) and (8.5) we arrive to the condition

$$
\left\langle O_{1 n}\right\rangle=\int_{-1}^{1}(1+t)^{\frac{p_{0}-q}{q}} L_{\frac{s+1}{2}}(t) P_{\frac{s-n}{2}}^{\left(0, \frac{p_{0}-q}{q}\right)}(t) d t=0,
$$


in the case where $s$ is odd and $n$ is odd and greater than 1 . And

$$
\left\langle O_{1 n}\right\rangle=\int_{-1}^{1}(1+t)^{\frac{p_{0}}{q}} L_{\frac{s}{2}}(t) P_{\frac{s-n-1}{2}}^{\left(0, \frac{p_{0}}{q}\right)}(t) d t=0,
$$

in case where $s$ is even and $n$ is odd and greater than 1 . Here we introduced the polynomial $L_{n}(t)$

$$
\frac{\partial S^{(0)}}{\partial v_{p_{0}}}(t)=L_{\frac{s+1}{2}}(t)
$$

for $s$ odd,

$$
\frac{\partial S^{(0)}}{\partial v_{q-p_{0}}}(t)=(1+t)^{\frac{p_{0}}{q}} L_{\frac{s}{2}}(t)
$$

for $s$ even.

Taking to the account these equations, the order of the polynomials $\frac{\partial S^{(0)}}{\partial v_{p_{0}}}$ and $\frac{\partial S^{(0)}}{\partial v_{q-p_{0}}}$ and the string equations (7.8), (7.9) we obtain the following explicit expressions

$$
\frac{\partial S^{(0)}}{\partial v_{p_{0}}}=\frac{p+q}{q}\left(P_{\frac{s+1}{2}}^{\left(0, \frac{p_{0}-q}{q}\right)}(t)-P_{\frac{s-1}{2}}^{\left(0, \frac{p_{0}-q}{q}\right)}(t)\right)
$$

if $s$ is odd and

$$
\frac{\partial S^{(0)}}{\partial v_{q-p_{0}}}=\frac{p+q}{q}(1+t)^{\frac{p_{0}}{q}}\left(P_{\frac{s}{2}}^{\left(0, \frac{p_{0}}{q}\right)}(t)-P_{\frac{s-2}{2}}^{\left(0, \frac{p_{0}}{q}\right)}(t)\right)
$$

if $s$ is even.

\section{Conclusions}

In this paper we have described the relation between the approach to $(p, q)$ models of Minimal Liouville gravity based on the Douglas string equation, on one hand, and the Frobenius manifolds of $A_{q-1}$ type on the other. As a result of this relation the generating function of correlation numbers in MLG is represented by the logarithm of the tau-function of the corresponding integrable hierarchy. All necessary information is encoded in the solution of the Douglas string equation and in the resonance relations between the parameters of the integrable hierarchy and the coupling constants of MLG. Using this relation and some special properties of the flat coordinates on the Frobenius manifold, we have found the appropriate solution of the Douglas string equation. This result generalizes analogues result found recently for Unitary models of Minimal Liouville gravity [6]. We have shown that the appropriate solution is consistent with the basic requirements of the conformal selection rules arising on the levels of one- and two-point correlation functions. Namely, the number of the parameters of the resonance transformations is exactly the number of the constraints following from the selection rules. Resolving these constraints we have found explicit form of the resonance transformations in terms of Jacoby polynomials. It would be interesting to investigate if this matching persists for multi-point correlation functions when the fusion rules of the underlying minimal models of CFT should be taken into account. This analysis requires also knowing the explicit form of the structure constants of 
the Frobenius algebra in the flat coordinates. We plan to study these questions in the near future. Another possible extension of our study is to consider different generalizations of the Minimal Liouville Gravity in the context of the Douglas string equation approach and its relations with different types of Frobenius manifolds. In particular, it would be interesting to understand what kind of the Frobenius manifold is relevant for $W_{N}$ Minimal Liouville gravity.

\section{Acknowledgments}

We thank Boris Dubrovin, Michael Lashkevich, Yaroslav Pugai and Grisha Tarnopolsky for useful discussions. The research was performed under a grant funded by Russian Science Foundation (project No. 14-12-01383).

Open Access. This article is distributed under the terms of the Creative Commons Attribution License (CC-BY 4.0), which permits any use, distribution and reproduction in any medium, provided the original author(s) and source are credited.

\section{References}

[1] A.M. Polyakov, Quantum Geometry of Bosonic Strings, Phys. Lett. B 103 (1981) 207 [INSPIRE].

[2] M.R. Douglas, Strings in Less Than One-dimension and the Generalized $K^{-} D^{-} V$ Hierarchies, Phys. Lett. B 238 (1990) 176 [InSPIRE].

[3] G.W. Moore, N. Seiberg and M. Staudacher, From loops to states in $2-D$ quantum gravity, Nucl. Phys. B 362 (1991) 665 [inSPIRE].

[4] A.A. Belavin and A.B. Zamolodchikov, On Correlation Numbers in 2D Minimal Gravity and Matrix Models, J. Phys. A 42 (2009) 304004 [arXiv:0811.0450] [InSPIRE].

[5] A. Belavin, B. Dubrovin and B. Mukhametzhanov, Minimal Liouville Gravity correlation numbers from Douglas string equation, JHEP 01 (2014) 156 [arXiv:1310.5659] [INSPIRE].

[6] V. Belavin, Unitary Minimal Liouville Gravity and Frobenius Manifolds, JHEP 07 (2014) 129 [arXiv: 1405.4468] [INSPIRE].

[7] A.A. Belavin, A.M. Polyakov and A.B. Zamolodchikov, Infinite Conformal Symmetry in Two-Dimensional Quantum Field Theory, Nucl. Phys. B 241 (1984) 333 [InSPIRE].

[8] V.A. Kazakov, A.A. Migdal and I.K. Kostov, Critical Properties of Randomly Triangulated Planar Random Surfaces, Phys. Lett. B 157 (1985) 295 [INSPIRE].

[9] V.A. Kazakov, Ising model on a dynamical planar random lattice: Exact solution, Phys. Lett. A 119 (1986) 140 [inSPIRE].

[10] V.A. Kazakov, The Appearance of Matter Fields from Quantum Fluctuations of $2 D$ Gravity, Mod. Phys. Lett. A 4 (1989) 2125 [inSPIRE].

[11] M. Staudacher, The Yang-lee Edge Singularity on a Dynamical Planar Random Surface, Nucl. Phys. B 336 (1990) 349 [InSPIRE].

[12] E. Brézin and V.A. Kazakov, Exactly Solvable Field Theories of Closed Strings, Phys. Lett. B 236 (1990) 144 [INSPIRE]. 
[13] M.R. Douglas and S.H. Shenker, Strings in Less Than One-Dimension, Nucl. Phys. B 335 (1990) 635 [INSPIRE].

[14] D.J. Gross and A.A. Migdal, Nonperturbative Two-Dimensional Quantum Gravity, Phys. Rev. Lett. 64 (1990) 127 [INSPIRE].

[15] I. Krichever, The Dispersionless Lax equations and topological minimal models, Commun. Math. Phys. 143 (1992) 415.

[16] B. Dubrovin, Integrable systems in topological field theory, Nucl. Phys. B 379 (1992) 627 [INSPIRE].

[17] R. Dijkgraaf, H.L. Verlinde and E.P. Verlinde, Topological strings in d $\mathscr{E} l t$; 1, Nucl. Phys. B 352 (1991) 59 [INSPIRE].

[18] V.G. Knizhnik, A.M. Polyakov and A.B. Zamolodchikov, Fractal Structure of $2 D$ Quantum Gravity, Mod. Phys. Lett. A 3 (1988) 819 [inSPIRE].

[19] P.H. Ginsparg, M. Goulian, M.R. Plesser and J. Zinn-Justin, $(p, q)$ string actions, Nucl. Phys. B 342 (1990) 539 [INSPIRE].

[20] P. Di Francesco and D. Kutasov, Unitary minimal models coupled to $2-D$ quantum gravity, Nucl. Phys. B 342 (1990) 589 [InSPIRE]. 